\title{
Advantages of Dextrose, Platelet Rich Plasma and Stem Cells over the Traditional Conventional Methods in Treatment of Sports Injuries and Joint Osteoarthritis
}

\author{
Dina Mohamed Ibrahim Soliman \\ 1. Department of Physical Medicine Rheumatology and Rehabilitation, Ain Shams University, Cairo 11566, Egypt \\ 2. Board certified in Regenerative Medicine, American Academy of Regenerative Medicine, Denver 80401, USA
}

\begin{abstract}
Osteoarthritis (OA) reflects a slowly evolving joint disease with loss and erosion of articular cartilage, osteophytes formation and weakness or laxity of the soft tissues in the synovial joints, particularly the large weight-bearing joints. The prevalence of the OA increases with age, and its radiological changes can be observed in over $50 \%$ of the retired people. Symptoms can vary from minimal to severe pain, stiffness, locking, decreased function and sometimes joint effusion. Inevitably, a wide range of potential therapies have been advocated, and not necessarily with strong supportive evidence. The Standard-of-care for treatment of pain is multidisciplinary; however, a recent systematic review reported no clear benefit of any one therapy. The Agency for Healthcare Research and Quality has called for the development of new therapies for pain management. In Interventional Regenerative Orthopedic Medicine (IROM), Dextrose is used as a tissue proliferant for treatment of OA. It has different ways of action aiming to do tissue regeneration. Platelet Rich plasma is also used in IROM and is very promising because of delivering "growth-factors" which stimulate the local tissue progenitor cells (mesenchymal stem cells) enhancing the regeneration of the tissues. Adult Mesenchymal stem cells is the hot topic nowadays in the non-surgical joint reconstruction. The results revealed that patients treated for sports injuries and joint OA using IROM (dextrose, PRP and stem cells) showed a greater improvement regarding their sports performance as well as joint pain and function compared to those treated with traditional methods (different modalities of physiotherapy, steroids injections.....etc). Moreover, athletes with sports injuries treated with steroid injections showed a deterioration in their sports performance, muscle strain, joint pain and function.
\end{abstract}

Key words: Injuries, pain, Non-surgical, Lyftogt PIT, prolotherapy.

\section{Introduction}

Osteoarthritis (OA) is a group of degenerative joint diseases with mechanical abnormalities. There are a variety of underlying factors for OA: Hereditary, developmental, metabolic, and mechanical deficits that may initiate the processes leading to OA.

Over the years, the pain management and rehabilitation modalities for musculoskeletal pain and OA have always been consisted in (1) non-pharmacological treatments, (2) pharmacological treatments, (3) surgical management and (4) non-surgical management.

Corresponding author: Dina Mohamed Ibrahim Soliman, MS.c. in PMR, PhD 1st part in PMR.

\subsection{Non-pharmacological Treatments}

Non-pharmacological treatments: Self-Management Education Programs "SMEPs", self-education and life style modification: weight loss, rest and low impact aerobic fitness exercises recommended by the American Association of Orthopedic Surgeons "AAOS" [1], use of shock-absorbing shoes with insoles and mechanical support devices. These conservative measures can provide an average of $20 \%$ more pain relief when compared to NSAIDs alone in patients with OA [2]. Rest, Ice, Compression and Elevation (RICE) are no longer recommended as all stop blood flow and inhibit healing [3].

The Physical Therapy: different studies reported that 
physical modalities have shown either a short term pain relief or ineffectiveness in treating $\mathrm{OA}$, or no significant difference between patients treated with physiotherapy and placebo group. These modalities are: therapeutic ultrasound, extracorporeal shock wave therapy, neuro-muscular electrical nerve stimulation, short wave diathermy, electro-magnetic field, laser therapy, trancutaneous electrical nerve stimulation, interferential currents and thermotherapy [4-6].

Bracing and Orthoses: American Association of Orthopedic Surgeons was unable to make a recommendation for or against the use of a brace for OA patients.

\subsection{Pharmacological Treatments}

Pharmacological treatments: Systemic medications, topical applications, intra-articular injections, chondroprotectors and other supplements.

Sytemic medications such as acetaminophens (paracetamol) that are the oral analgesic of choice for relieving the pain of OA because they are relatively safe and generally well tolerated. Non-steroidal anti-inflammatory drugs have adverse drug reactions related to gastrointestinal tract, kidneys, liver, $20 \%$ of congestive heart failure, and $80 \%$ of myocardial infarction. Oral steroids (corticosteroids) are not recommended in the treatment of musculoskeletal pain and OA because of their modest benefit and high rate of adverse effects on long-term use such as hyperglycaemia, insulin resistance, diabetes mellitus, hypertension, osteoporosis, hypogonadism, hypothyroidism, amenorrhea, erectile dysfunction, cataract, retinopathy, anxiety, depression and colitis. Opioids have small to moderate beneficial effects for patients with musculoskeletal pain and OA, and these effects are outweighed by large increases in the risk of adverse events, therefore they should not be routinely used.

Chondroprotectors are also used in the treatment of OA. The Agency for Healthcare Research and Quality has concluded that glucosamine products administration showed no significant difference compared to placebo.

Other supplements that might be useful in treatment of OA: Ginger, bromelain, protease enzyme extracted from pineapple, avocado, vitamins such as B9, B12, D3 and $\mathrm{E}$.

Topical applications as topical NSAIDs and capsaicin preparation, the later are not recommended to be applied any more [7] as they act by subsiding the neurogenic inflammation after their initial stimulation.

Intra-articular injections of viscosupplementation (hyaluronic acid) e.g., Synvisc and Euflexxa for OA are minimally effective. The predominant mechanism of hyaluronic acid is unknown, and no study has demonstrated that these drugs alter the disease course.

Although intra-articular injections of steroids inhibit the accumulation of inflammatory cells, corticosteroids lead to a decrease in bone, ligament and tendon strength. They inhibit the synthesis of proteins, collagen, proteoglycans particularly the articular cartilage by inhibiting chondrocyte production, and they inhibit angiogenesis. The result is weakened synovial joints, and this weakness increases the pain. Increased pain leads to more steroids injections [8], therefore these drugs should play no role in sports injury care because despite of reducing the pain, they do so at the cost of destroying the tissues.

A systematic review found a significant short-term reduction in pain and swelling for patients with intra-articular corticosteroids injections compared with placebo injections, however good evidence of long-term benefit and exact efficacy remain controversial. Another study revealed no significant difference in rate of joint space narrowing between the two groups, and reported no case could be made for a disease-modifying effect of corticosteroids injections $[1,9]$.

\subsection{Surgical Management}

Surgical management and arthroscopic surgery is not an appropriate treatment for joint OA. Two 
well-designed randomized controlled trials of arthroscopic surgery for treatment of knee OA showed no benefit. Surgery in general is not without complications, the most common risk of surgery is anesthesia and the reaction to anesthesia or other drugs. The most common side effects of spine surgeries are the failure to relieve the pain symptoms following the surgery or recurrent disc herniation. Nerve damage which can result in weakness, paralysis, pain, sexual dysfunction, loss of bowel or bladder control can also occur.

\subsection{Non-surgical Management}

Non-surgical Interventional Regenerative Orthopedic Medicine "IROM" (prolotherapy and Lyftogt perineural injection therapy). Being under the umbrella of IROM, prolotherapy "proliferative regenerative therapy", is a method of injection treatment designed to stimulate healing. It is a fundamental and an effective injection therapy for the repair of the injured fibrous connective tissue [10] that hold our skeletal infrastructure together, including the ligaments, tendons, muscle fascia, joint capsular tissue and cartilage on the inside of joints. It is based on the premise that chronic musculoskeletal pain is due to the inadequate repair of the fibrous connective tissue (connective tissue insufficiency) resulting in ligament and tendon weakness or relaxation (laxity) [11].

Prolotherapy has been used in a form recognizable to contemporary practitioners for at least 75 years. Literature from 1930's to 2000's reported positive clinical outcomes and that it is the treatment of choice for musculoskeletal injuries and OA especially if the patient fails to improve after physical therapy, chiropractice or orthopathic manipulation, steroid injections, radiofrequency denervation or surgical interventions or if such modalities are contraindicated [12].

It was around 400 years before Jesus crisis when Hippocrates, father of modern medicine, said that healing is a matter of time but sometimes is a matter of opportunity $[11,13]$. Dr. George S. Hackett, father of prolotherapy, was the one who developed the prolotherapy technique, formalized its protocol and he was pioneer at. In 1940 he coined the term Prolotherapy and described it saying that it stimulates the production of new fibrous tissue and bone cells that will strengthen the weld at the fibro-osseous junction [11, 14].

The collagen fibers of the periosteum are normally arranged in parallel to the bone surface except at the ligament and tendon attachments "fibro-osseous junction", they traverse the bone tissue obliquely and are referred to as (Sharpey's fibers) where the calcium molecules of the bone encroach on the collagen fibers of the ligaments and tendons. At these fibro-osseous junctions, there are the greatest stresses and most of the injuries of the soft tissues stabilizing and moving the joints occur at these junctions [13].

The blood supply for long bones is dependent upon periosteal vessels that penetrate the marrow cavity. At the fibro-osseous junctions, the dense connective tissue of the ligaments and tendons is not well supplied with vascular tissue which makes the injuries of these tissues hard to heal. When the tensile strength is deficit and not corrected, the connective tissue goes into stretching when they are bearing loads.

In 1953, Gardner and Gray discussed the abundant supply of sensory nerves in the ligaments, tendons and the capsules of the joints and they reported that the most effective stimulus to these nerves is in twisting the joints and stretching of the lax structures. Pain is perceived when normal tension on a ligament stretches the relaxed ligament fibers, resulting in stimulation of the sensory nerves as the nerve fibers do not stretch [ 8 , $11,13,15]$.

The most important molecule in initiating and maintaining pain related behavior in animals and pain experience in humans is the transient receptor potential vanilloid type 1 (TRPV1) that was previously called Capsaicin receptor. These molecules are located on the nervi-nervorum of the peripheral nervous system; they 
are non-specific membrane cation channels allowing the influx of $\mathrm{Na}+$ and $\mathrm{Ca} 2+\mathrm{Na}+$ influx results in spike formation and increased action potential causing the neuropathic pain, and $\mathrm{Ca}+$ influx triggers the release of the neuropeptides such as Substance P "SP" and Calcitonin Gene Related Peptide "CGRP", Nitric Oxide "NO", Vasoactive Intestinal Polypeptide "VIP", Neurokinin A "NKA" and 5-hydroxytriptamine "5-HT" causing the neurogenic inflammation [11].

Hilton's law 1879 stated that the nerve supplying a joint also supplies the muscles that move the joint and the skin covering the joint, so treating the cutaneous inflamed nerves supplying the skin over the joint will eventually treat the nerves supplying the joint and the nerves supplying the muscles moving the joint $[7,11]$. This is the Lyftogt perineural injeciton treatment.

Prolotherapy works by causing a temporary, low grade inflammation at the fibro-osseous junctions to activate the fibroblasts to the injury area, to synthesize precursors for mature collagen by raising the level of growth factors $[11,16]$. The ideal proliferating solution is one that has the following desirable features: (1) A minimum of discomfort, (2) a minimum of early inflammatory exudates, (3) a maximum of permanent white fibrous connective tissue, (4) no systemic reaction, (5) no sloughing or destruction of tissue [11]. In addition, the proliferant solutions have been hypothesized to stimulate the normal flow of nerve growth factors and subsequently they allow tissue and nerve repair [4].

The proliferants used in IROM are: (1) Chemical irritants, (2) particulates, (3) osmotic agents, (4) chemotactic agents, (5) platelet rich plasma, and (6) adult mesenchymal stem cells.

The osmotic agent (glucose or dextrose) is the most popular proliferant used. Glucose acts by more than one mechanism as follows: (1) Osmotic shock (glucose 15\%) which acts by dehydrating the cells at the injection site causing a net flow of fluid across the semipermeable cell membrane from intracellular to extracellular fluid releasing the cellular fragments (proteins and membrane fragments) attracting the granulocytes. Upsetting the cell membrane and the delicate balance of these living cells, puts the arachidonic acid pathway on fire [17]. (2) Concentrated glucose provides a gradient of a desirable nutrient which attracts mobile cells of the body's repair apparatus to the injection site [17]. (3) Glucose 5\% leads to a rapid reduction of pain within 10-20 seconds and gives an immediate analgesia that can last from 4 hours to 3 weeks. It blocks the TRPV1 ion channels and ends up by a down-regulation of these molecules [18]. (4) Genes for growth factor production are activated within 20 minutes after exposure of the human cells to $0.45 \%$ to $0.6 \%$ glucose (the normal cell has $0.1 \%$ to $0.3 \%$ glucose in the intra and extracellular spaces) [2].

Platelet Derived Growth Factors have a major role in stimulating the progenitor mesenchymal stem cells to produce different types of cells. Research studies and clinical practice have shown PRP therapy to be very effective at relieving pain and returning patients to their normal lives. Both ultrasound and MRI images have shown definitive tissue repair after PRP therapy, confirming the healing process.

Stem cells have the remarkable potential to develop into many different cell types. They are derived either from the bone marrow of from the adipose tissue, and they serve as a sort of internal repair system dividing to replenish other cells. Stem cells showed great results in the treatment of musculoskeletal pain and degenerative diseases as they stimulated the formation of all the different tissues of the body: muscle, cartilage, tendon, ligament, bone, blood, nerve, etc....

\section{Methods}

More than 300 patients have been treated for their musculoskeletal pain and OA over the last three years with the following methods: (1) Non-pharmacological treatments (physiotherapy). (2) Pharmacological treatments (intra-articular injections of viscosupplements and steroids). (3) Non-surgical 
Interventional Regenerative Orthopedic Medicine "IROM": a. Prolotherapy (dextrose, PRP and bone marrow adult mesenchymal stem cells); b. Lyftogt Perineural Injection Therapy.

The assessment was done using Visual Analogue Scale (pain) and Western Ontario and McMaster Universities osteoarthritis index (function) [19]. This was done for every patient before the start of the treatment, and on each time the patient came to get the treatment session (interval between the sessions depends on the type of treatment used for the patient).

\section{Results and Conclusions}

Patients with musculoskeletal pain and OA of different joints in the body treated with physiotherapy did not get the satisfying improvement regarding the decrease in VAS and the increase in function.

Some of the patients treated with intra-articular injections of viscosupplements showed a slight pain relief and a slight increase in function, then they went back again to their level of pain and disability they started at. Others did not get any improvement at all.

Although patients treated with steroids intra-articular injections showed an immediate pain relief as well as an increase in function, after 3 months or even less they showed a worsening in their condition and there onwards their pain became a far worse than before and the function was decreased.

Athletes and patients treated with intra-articular and local steroids injections over a long period of time showed many systemic side effects especially endocrinological complications affecting all their body systems, healing, weight, and sports performance. They never did get back to play with their normal endurance, strength and performance.

Patients who underwent surgeries most probably had to go through the surgical procedures again, either in the same region or in another areas due to the creation of new extra-stresses in the body after the surgery. These new additional stresses are resulted from the changes in the internal line of body pressure after the surgical procedure. This is due to the changes in the center of gravity of the body part subjected to surgery, subsequently other areas will be vulnerable to injuries. Accordingly, the body adapts a new abnormal biomechanical pattern to accommodate the new body situation in order to enable its movement, which puts the musculoskeletal system in a vicious circle of pain re-generation, and the patient never goes back to normal.

Non-surgical Interventional Regenerative Orthopedic Medicine "IROM" is the bridge to the future. It helps in regenerating and reconstructing the tissues and the joints without changing neither the stress distribution of the line of pressure nor the biotensegrity of the body. Biotensegrity is defined by the dependence of the different body parts on each other. These parts work simultaneously with each other (compression-tension), which makes the different body parts handle different movement.

Lyftogt Perineural injection Treatment is the superficial injection technique of IROM. It is the challenging ease that alleviates the pain of the patient immediately by blocking the TRPV1 molecules, and hence decreases the neurogenic inflammation, the neuropathic pain and the muscle spasm, therefore increases the function.

Prolotherapy treatment for musculoskeletal pain and joints is the deep technique of IROM. It is a line of conservative treatment that gives positive and promissing results for the patients.

Prolotherapy improves tissue healing and hence improves the articular cartilage, ligament and tendon repair. Consequently, it decreases the pain (VAS) and increases the function (WOMAC).

Using platelet rich plasma and adult mesenchymal stem cells as proliferative solutions in prolotherapy technique for treatment of musculoskeletal pain and joints gives the patients the quicker and best results regarding the pain and function. However, the use of the adult mesenchymal stem cells in this technique gives the best results even with the most severe and late 
cases.

\section{References}

[1] Rozendaal, R., Uitterlinden, E., Osch, G., Garling, E., Willemsen, S., Ginai, A., Verhaar, J., Weinans, H., Koes, B., and Bierma, S. 2009. "Effect of Glucosamine Sulphate on Joint Space Narrowing, Pain and Function in Patients with Hip Osteoarthritis; Subgroup Analyses of a Randomized Controlled Trial." Osteoarthritis Cartilage 17 (4): 427-32.

[2] Riskowski, J., Dufour, A., and Hannan, M. 2011. "Arthritis, Foot Pain \& Shoe Wear: Current Musculoskeletal Research on Feet." Curr. Opin. Rheumatol. 23 (2): 148-55.

[3] Struijs, P., Blankevoort, L., Welling, L., Dijk, C., and Kerkhoffs, G. 2012. "What Is the Evidence for Rest, Ice, Compression, and Elevation Therapy in the Treatment of Ankle Sprains in Adults?" J. Athl. Train 47 (4): 435-43.

[4] Adam, D. 2011. "Neural Prolotherapy." Journal of Prolotherapy 3 (2): 639-43.

[5] Ainsworth, R. 2007. "A Prospective Double Blind Placebo-Controlled Randomized Trial of Ultrasound in the Physiotherapy Treatment of Shoulder Pain." Rheumatology 46 (5): 815-20.

[6] Saunders, J., Hungerford, B., Wisbey-Roth, T., Lucas, P., and Wilson, S. 2010. "The Use of Prolotherapy in the Sacro-Iliac Joint.” Br. J. Sports Med. 44 (2): 100-4.

[7] Rabago, D., Jeffery, J., Marlon, M., Richard, K., Jessica, G., Neil, A., and Aleksandra, Z. 2013. "Dextrose Prolotherapy for Knee Osteoarthritis: A Randomized Controlled Trial." Annals of Family Medicine 11 (3): 229-37.

[8] Hauser, R., and Hauser, M. 2004. Prolo Your Pain Away. Oak Park Illinois: Beulah Land Press, 42.

[9] Lyftogt, J. 2007. "Subcutaneous Prolotherapy Treatment of Refractory Knee, Shoulder and Lateral Elbow Pain." Aust. Musculoskeletal Med. 12 (2): 110-2.

[10] Soliman, D. 2016. Pain Management by Prolotherapy and Perineural Injection Therapy. Germany: Lambert Academic Publishing, 13-38.

[11] Hackett, G. 1960. "Prolotherapy in Whiplash and Lowback Pain.” Postgraduate Medicine 27 (2): 214-9.

[12] Reeves, K., and Hassanein, K. 2000. "Randomized Prospective Placebo Controlled Double Blind Study of Dextrose Prolotherapy for Osteoarthritic Thumbs and Finger (DIP, PIP and Trapeziometacarpal Joints): Evidence of Clinical Efficacy." J. Altern. Complement Med. 6 (4): 311-20.

[13] Hauser, R. 2009. "The Deterioration of Articular Cartilage in Osteoarthritis by Corticosteroid Injections." Journal of Prolotherapy 1 (2): 107-23.

[14] Hepper, C., Halvorson, J., Duncan, S., Gregory, A., Dunn, W., and Spindler, Kurt. 2009. "The Efficacy and Duration of Intra-Articular Corticosteroid Injection for Knee Osteoarthritis.” J. Am. Acad. Orthop. Surg. 17 (10): 638-46.

[15] Alderman, D. 2007. "Prolotherapy for Musculoskeletal Pain.” Practical Pain Management 7 (1): 10-4.

[16] Richmond, J., Hunter, D., Irrgang, J., and Jones, M. 2009. "Treatment of Osteoarthritis of the Knee (Non-Arthroplasty)." J. Am. Acad. Orthop. Sur. 17 (9): 591-600.

[17] Banks, A. 1991. "A Rationale for Prolotherapy.” Journal of Orthopedic Medicine 13 (3): 1-10.

[18] Lyftogt, J. 2010. "Neural Prolotherapy." Presented at the Hackett Hemwall Foundation and the Italian Society for Prolotherapy, Ferrara, Italy.

[19] Bellamy, N., Bell, M., Goldsmith, C., Pericak, D., Walker, V., Raynauld, J., Torrance, G., Tugwell, P., and Polisson, R. 2005. "Evaluation of WOMAC 20, 50, 70 Response Criteria in Patients Treated with Hylan G-F 20 for Knee Osteoarthritis.” Ann. Rheum. Dis. 64 (6): 881-5. 